\title{
CONSIDERACIONES ACTUALES SOBRE EL USO DE FÉRULAS OCLUSALES EN REHABILITACIÓN ORAL: UNA REVISIÓN CRÍTICA
}

\author{
CURRENT CONSIDERATIONS ABOUT THE USE OF OCCLUSAL SPLINTS IN ORAL \\ REHABILITATION: A CRITICAL REVIEW \\ Anny Valeria Chalco Valdivia ${ }^{1}$ \\ annycita1984@hotmail.com \\ ORCID: 0000-0002-9935-521X \\ Ana Isabel López Flores ${ }^{1}$ \\ dra.lopz@gmail.com \\ ORCID: 0000-0002-1104-2439
}

\section{RESUMEN}

El uso de las férulas oclusales en la actualidad, en el vasto campo de la odontología, es de gran importancia para el tratamiento de trastornos témporo mandibulares (TTM), así como en el tratamiento de otras para funciones relacionadas con la articulación témporo mandibular (ATM). Actualmente, existe variedad en cuanto al diseño de férulas oclusales, hechas con diferentes materiales, con diferentes resistencias y utilizadas para cada tipo de disfunción temporomandibular que se presente, así como para las parafunciones existentes. Dentro de esta variedad son solo cinco tipos de férulas las que se conocen ampliamente, según la evidencia científica. Cada una de ellas tiende a considerar diversas características en la forma de fabricación y sus indicaciones; así mismo, el clínico debe ejecutar una buena elección, un plan de control adecuado para cada caso, con un diagnóstico acertado que llevará al éxito de los resultados. Se debaten diferentes aspectos en cuanto el uso de las férulas en el tratamiento de rehabilitación oral, incluyendo su uso para desprogramar la ATM, correcciones en la relación cóndilofosa, eliminación de desarmonías oclusales para aliviar el dolor en la ATM, su influencia en la eficiencia muscular y su efecto en la carga muscular. Se debe diseñar un tipo de férula para cada trastorno en específico y evaluar su verdadero valor terapéutico. En esta revisión crítica, se busca describir y analizar el uso de las férulas oclusales, sus principales funciones y aplicaciones en pacientes con diferentes trastornos.

Palabra clave: férulas oclusales, articulación témporo mandibular, bruxismo, trastorno témporo mandibular

\section{ABSTRACT}

At present, the use of the occlusal splints in the vast field of dentistry is of great importance for the treatment of temporo mandibular disorders (TMD) as well as in the treatment of other functions related to the temporo-mandibular joint (ATM).

Citar como: Chalco-Valdivia AV, López-Flores Al. Consideraciones actuales sobre el uso de férulas oclusales en rehabilitación oral. Una revisión crítica. Rev Cient Odontol (Lima). 2019; 7 (1): 157-167.

${ }^{1}$ División de Rehabilitación Oral, Carrera de Estomatología, Universidad Científica del Sur. Lima, Perú. 
The design of occlusal splints varies, involving different materials, different resistances and according to the type of temporomandibular dysfunction as well as existing parafunctions. To date 5 types of splints have been described, each of which considers different characteristics related to manufacturing and their indications. Likewise, to ensure adequate results, clinicians must select the splint to be used according to the characteristics of each patient. We discuss different aspects such as the use of splints in the treatment of oral rehabilitation, including their use to deprogram TMD, corrections in the condyle-fossa relationship, elimination of occlusal disharmony to relieve TMJ pain, the influence of splints on muscle efficiency and their effect on muscle load. Splints must be designed individually to assess their real therapeutic value, taking into account the specific conditions of each patient. This critical review, seeks to describe and analyze the use of occlusal splints and their main functions and applications in patients with different oral disorders.

Keywords: splints, occlusal temporomandibular joint, bruxism, temporomandibular disorder

\section{INTRODUCCIÓN}

Las férulas oclusales son denominadas también aparatos interoclusales, placas o planos oclusales, y son artificios removibles que en su manejo suelen ser conservadoras, poco invasivas y reversibles $\left(^{1-9}\right)$. Son, además, dispositivos que los clínicos utilizan para el manejo en el tratamiento de trastornos témporo mandibulares (TTM) y parafunciones con el fin de mejorar los problemas del paciente en la cavidad oral. Estos dispositivos intraorales, gracias a un buen diseño, cumplen aceptablemente funciones como las de establecer el equilibrio neuromuscular en el sistema estomatognático a nivel de la articulación témporo mandibular (ATM), con relación a los tendones, el disco articular, entre otros. El dolor en la región miofascial está relacionado en un alto porcentaje con los TTM y se localiza con mayor frecuencia en la ATM y en el área preauricular de los músculos masticatorios $\left({ }^{10}\right)$. El uso de férulas oclusales resulta ser un medio efectivo para aliviar y aminorar este dolor.

De los tipos de férulas, las de estabilización oclusal se vienen usando como dispositivos que tienen un efecto terapéutico positivo sobre los TTM; según la literatura revisada, aun así, se deben realizar estudios relacionados con la eficacia de dichos artificios en el tratamiento de pacientes con problemas de TTM, utilizando protocolos de manejo y controles periódicos de los dispositivos. El uso de placas o férulas se inicia con relación a la etiología del dolor, la cual es de carácter multifactorial y tiene a la psicología del paciente como un factor influyente. Además, el tipo de trauma ocasionado en la ATM puede haber ocasionado un cambio en su anatomía, lo que varía el curso del diagnóstico. Según las estadísticas, las mujeres son las más susceptibles a ser usuarias de las placas o férulas oclusales, entre las edades de 12, 20 y 40 años, pues se ven más afectadas por el tema dolor y su recuperación se hace más compleja ${ }^{(11-13)}$.

Aún hacen falta muchos estudios con respecto a este tema; sin embargo, realizar una revisión crítica de la literatura actual es importante. Por tanto, el objetivo del estudio fue orientar un buen diagnóstico de los TTM y otras parafunciones, y de esta manera ayudar a que se realice una buena elección del tipo de férulas oclusales para pacientes con alteraciones témporo mandibulares. Asimismo, permitirá analizar, mediante el seguimiento del paciente, si tienen un efecto positivo en el tratamiento terapéutico del dolor a corto o largo plazo $\left({ }^{14,15}\right)$, 
además de conocer su mecanismo de acción y otras formas de tratar los TTM y las parafunciones.

\section{EVALUACIÓN DE TIPOS DE FERULAS OCLUSALES EN PACIENTES CON TTM Y BRUXISMO}

Las férulas oclusales, como parte de su función, generan una reducción significativa en los signos y síntomas de las parafunciones y los TTM; por lo tanto, son herramientas usadas de forma terapéutica por el profesional a cargo. Su éxito clínico se mide en un $70 \%$ a un $90 \%$ en la disminución del dolor $\left({ }^{16-21}\right)$, en especial del miofascial ${ }^{22}$. Se clasifican de acuerdo con su funcionamiento, a partir del reposicionamiento mandibular y la relajación de los músculos. Su propósito terapéutico principal es modificar el estado condilar y, en cuanto a la cobertura, puede ser total y parcial, según su grado de resistencia: dura, rígida, semirrígida y resiliente $\left({ }^{11}\right)$. En la actualidad, existen diferentes tipos de férulas que son objeto de análisis en rehabilitación oral, entre ellas las de estabilización, para mordida anterior y pivotante, entre otras $\left({ }^{22}\right)$, siendo utilizadas con mayor frecuencia las férulas de reposicionamiento anterior y de estabilización $\left({ }^{23,24}\right)$.

Las férulas permisivas o de estabilización tienen como propósito dirigir al cóndilo a la ubicación más estable, es decir, en posición céntrica; son temporales y removibles $\left({ }^{25-26}\right)$, y a través de ellas la actividad muscular normal disminuye para encontrar un aceptable equilibrio neuromuscular $\left({ }^{26-27}\right)$. Poseen diferentes diseños, entre los que están las férulas blandas, las neuromiorrelajantes y también las que son solo miorrelajantes, como las de tipo Michigan. Son indicadas para la estabilización de la hiperactividad muscular, mediante la reducción de la actividad parafuncional que se produce por momentos de estrés, y también están indicadas en casos de retrodiscitis postrauma. Estas férulas se fabrican con acrílico duro. En cuanto a su ubicación, es preferible que sea en el maxilar superior, porque cubre la mayor cantidad de tejidos, es más estable y la vulnerabilidad a quebrarse es menor $\left({ }^{12-15}\right)$.

La férula de posicionamiento anterior o férula directria tiene como función principal tratar los trastornos de alteración a nivel discal, sobre todo si hay luxación o adelantamiento, lo que produce ciertos ruidos en la apertura, generados precisamente por la posición alterada en que se encuentra. Estas férulas directrices llevan la mandíbula hacia una posición protusiva, con el fin de que el cóndilo mantenga una relación con el disco y evitar así que este quede por detrás del cóndilo. Se indica para tratar alteraciones a nivel del disco articular y resulta de gran utilidad en pacientes que presentan ruidos articulares y procesos de inflamación en el disco. Se fabrica con acrílico transparente. La ubicación en boca puede ser en ambos maxilares $\left({ }^{12-15}\right)$.

La férula de mordida anterior es un dispositivo cuyo desempeño en boca radica en producir la desoclusión de los dientes posteriores, separándolos de su participación dentro del sistema estomatognático. Se usa en pacientes con hábitos parafuncionales, pero su uso está limitado, ya que puede causar una sobreerupción en los dientes posteroinferiores cuando no presenta antagonista. Su uso no debe ser prolongado (máximo 15 días) y debe estar controlado por el cirujano dentista, de no ser así puede presentar complicaciones ya que el dispositivo solo cubre una parte del arco dental $\left({ }^{12-15}\right)$.

La férula de mordida posterior contribuye a reponer la posición mandibular y establecer la dimensión vertical. Se indica en casos en los que se quiere 
aumentar la dimensión vertical y reposicionar el cóndilo en su lugar; también puede ser utilizado en casos de retrodiscitis. El problema radica que solo cubre la parte posterior, lo que en su defecto también crea una sobreerupción de las piezas anteriores, intruyendo las piezas ocluidas. Este tipo de férulas pueden ser blandas o rígidas $\left.{ }^{12-15}\right)$.

En el caso de la férula pivotante, al existir una fuerza en sentido vertical debajo del mentón, los dientes anteriores tienden a juntarse, mientras que el cóndilo sufre una destrucción alrededor del punto de pivotación anterior. Se indica para reducir las presiones intraarticulares, siempre y cuando las fuerzas de la mandíbula se sitúen por delante del punto pivotante. Es utilizada en pacientes con osteoartritis de la ATM $\left.{ }^{12-15}\right)$.

La férula Niti (noiceptive inhibition of the trigeminal nerve) tiene como función principal el aminoramiento del dolor asociado al trayecto del disco con reducción. Este tipo de placa oclusal tiende a generar más ruido articular, factor por el cual su uso deber ser motivo de mayor investigación. Además, posee un punto de contacto único en la zona incisal, lo que da lugar al efecto de inhibición. De esta manera, los nocireceptores reciben la presión en los dientes incisivos e inhiben la contracción de los músculos elevadores. Se indica cuando hay dolor en la disfunción cráneo mandibular (DCM), bruxismo, cefaleas, músculos masticatorios y síndrome de SADAM, entre otros $\left({ }^{12-15}\right)$.

La férula blanda, resiliente o extraconfort, es un dispositivo flexible hecho con un material elástico, propiedad por la cual puede acomodarse fácilmente a los maxilares. El fin terapéutico es lograr el contacto de manera concomitante para desprogramar la ATM y aliviar alteraciones articulares y musculares. Se indica en pacientes con bruxismo, en deportistas - para evitar que el trauma sea de menor impacto, cuando estos se encuentran realizando sus actividades deportivas - y en pacientes con trauma en arcos dentarios $\left({ }^{12-15}\right)$.

La férula Pefe tiene como función principal contactar con los dientes posteriores para lograr el funcionamiento de ciertos músculos por un espacio de 15 horas. Al contactar con los dientes anteriores, se fracciona o rompe el engrama muscular, lo que genera la desprogramación muscular, y se cambia la información propioceptiva del cerebro. Se usa para la mayoría de las disfunciones anteriormente citadas $\left({ }^{(2-15)}\right.$.

\section{ANÁLISIS DEL COMPORTAMIENTO MECÁNICO DE LAS FÉRULAS OCLUSALES}

En la actualidad, se sabe que el mecanismo fisiológico de respuesta del organismo asociado al uso de férulas oclusales aún es controversial $(21,26-28)$. Sin embargo, es uno de los tratamientos en cuanto a la reducción del dolor miofascial se refiere, en especial por la respuesta positiva del paciente durante su uso; por ello, son utilizadas con frecuencia (21,26${ }^{28}$ ). Entre los mecanismos de acción que se han citado a lo largo del tiempo, se encuentra la reducción de la actividad en los músculos elevadores, la disminución del dolor miofascial, la reducción de ruidos periauriculares, la estabilidad del maxilar y la mandíbula, la desaparición temporal de las interferencias oclusales, el aumento de la dimensión vertical y, en algunos casos, el efecto placebo $\left({ }^{26-29,30}\right)$. También, elimina el riesgo de sobreerupción, la intrusión dentaria y, de esta manera, reduce el riesgo de adquirir otros síntomas añadidos y relacionados con los músculos de la ATM. 
TERAPÉUTICA DE LAS

FÉRULAS OCLUSALES

EN LA PREVENCIÓN Y

TRATAMIENTO DE LOS

TTM Y BRUXISMO

Existe diversidad de tratamientos para mejorar las condiciones de los TTM que pueden ser quirúrgicos o no. Por tanto, el diagnóstico acertado para el tratamiento dependerá absolutamente de las destrezas del clínico para el manejo y las ideas sobre su causa. La finalidad del uso de las férulas es aliviar el dolor miofascial, el ruido articular y recuperar la función mandibular normal, recientes estudios concluyen que aún no hay evidencia acerca de la efectividad de las férulas o placas oclusales, pero lo cierto es que sí se obtienen beneficios y buenos resultados en cuanto a evitar el desgaste de los dientes. El uso de las férulas mejora notablemente la calidad de vida del paciente y constituye una opción no invasiva. Su uso también puede relacionarse con el efecto placebo, así como con la relación doctor/paciente.

Una vez colocadas, las férulas deben tener un control siete días después. Si es necesario, se realizará un ajuste 0 , de lo contrario, se verificará si la oclusión ha permanecido estable $\left({ }^{31}\right)$. En los casos de pacientes que usan prótesis implanto soportadas, se debe tener un mayor cuidado, ya que no se puede controlar la mandíbula por la falta de receptores periodontales $\left({ }^{30}\right)$. Al modificar la oclusión por medio de tratamientos invasivos o agresivos, se producen daños colaterales y no influyen en una dimensión morfofisiológica real en el sistema estomatognático. Otros estudios han revelado que la terapia conductual, la acupuntura y los ejercicios mandibulares, acompañados por tratamientos farmacológicos, pueden resultar eficaces para aminorar el dolor en la ATM. Sin embargo, en cuanto a cirugías y pruebas electrofísicas, la evidencia es insuficiente ${ }^{\left({ }^{32}\right)}$. El dolor crónico que caracteriza los TTM se ha convertido en uno de los problemas más difíciles de solucionar para el clínico ( $\left.{ }^{30-36}\right)$ en la parte clínica el cirujano dentista aplica métodos combinados para obtener un mejor manejo de resultados y el éxito en dependencia de las necesidades del paciente.

Se debe educar al paciente, explicarle el motivo y la naturaleza benigna del trastorno, adiestrarlo en el autocuidado, explicarle que la función mandibular ya no es la misma; por lo tanto, se debe limitar el uso de gomas de mascar, evitar bostezos amplios y mantener una dieta blanda. Se deben modificar también hábitos presentes como morder objetos, bruxar, onicofagia, entre otros $\left({ }^{36-39}\right)$, se debe realizar masajes por 20 minutos cuatro veces al día, evitar la cafeína porque es estimulante, dormir en posición decúbito supino, evitar el estrés emocional y hacer seguimientos acompañado de visitas regulares $\left({ }^{40-42}\right)$.

La terapia conductual debe ser inducida por un profesional calificado que esté apto para realizar terapias de relajación muscular, retroalimentación, hipnosis y yoga $\left.{ }^{(43-46}\right)$. Este conjunto de actividades ayudará a que haya un incremento en el sistema nervioso simpático, la disminución del tono muscular, la ansiedad y el estrés $\left({ }^{42}\right)$. En algunas ocasiones los TTM se traducen como expresiones somáticas de alteraciones psicológicas y psiquiátricas, cuando esto sucede el paciente debe ser remitido al psiquiatra como parte de la estrategia del tratamiento $\left({ }^{40,45,46}\right)$.

En cuanto a la farmacoterapia, el grupo farmacológico de los aines son los de elección, pues su efectividad radica a nivel inflamatorio leve a moderado $\left({ }^{47}\right)$. Las drogas que mayormente se pueden utilizar son ibuprofeno, aspirina, piroxicam, naproxeno, entre otros $\left({ }^{48}\right)$. Por el momento, no existe un medicamento que 
pueda combatir y aliviar los TTM; por ello, es importante saber que la medicación deber ser por intervalos de tiempo, para evitar la resistencia al medicamento, y estar asociada a otras terapias.

La terapia física o fisioterapia es un procedimiento que involucra el movimiento de músculos específicos y sirve para aminorar el dolor mucoesqueletal. En la aplicación de esta modalidad se puede incluir masajes, movilización articular, ejercicios mandibulares y acupuntura. Sin embargo, si el paciente no puede autorrelajarse, se usará el biofeedback (terapia mecánica), que consiste en la colocación de electrodos que emiten un sonido y la intensidad con la que es transmitida es proporcional a la severidad de la hipertonicidad muscular $\left({ }^{48}\right)$. También se puede recurrir a estimulación eléctrica y eléctrica transcutánea, estimulación galvánica, ionoforesis, ultrasonidos, láser bandos que producen aumento de la bioestimulación tisular $\left({ }^{49,50}\right)$, láser helio neón y láser de diodos semiconductores.

Finalmente, se ha reportado que la terapia oclusal ayuda al alivio del dolor de los TTM en pacientes con predisposición, aunque no es un factor etiológico $\left({ }^{51-53}\right)$, y que la terapia quirúrgica es una alternativa, pero indicada en pequeños porcentajes y solo para desórdenes específicos $\left({ }^{54}\right)$. De acuerdo con el diagnóstico, las cirugías pueden ser abiertas o cerradas $\left({ }^{55-56}\right)$; la menisectomía se realiza en casos de disfunción y dolor articular, y la condilectomía se indica en casos de disfunción y dolor de larga data $(57,58)$.

\section{CONCLUSIONES}

- El diagnóstico es un proceso de gran importancia para poder elegir el tipo de férula y sus indicaciones. Se usará para contribuir en el bienestar del paciente.

- La evidencia científica señala la efectividad del uso de las férulas oclusales, en el tratamiento de síntomas y signos de TTM incluyendo patologías como el bruxismo.

- El mecanismo de acción de las férulas aún es controversial, puesto que algunas de ellas, colocadas en boca, producen ruidos articulares. Por tanto, se sugieren más estudios para contribuir a un mejor análisis sobre su uso.

Contribución del autor: Anny Valeria Chalco Valdivia y Ana Isabel López Flores participaron en la concepción del artículo, la recolección de datos, su redacción y aprobación de la versión final.

Fuente de financiamiento: Autofinanciada

Conflicto de Interés: las autoras declaran no tener conflictos de interés de ningún tipo. 


\section{REFERENCIAS BIBLIOGRÁFICAS}

1. Okeson JP. Tratamiento de oclusión y afecciones temporomandibulares. Madrid: Evolve, Elsevier; 2008.

2. Buescher JJ. Temporo mandibular joint disorders. Am Fam Physician. 2007; 76 (10): 1477-82.

3. Tanaka E, Detamore MS, Mercuri LG. Degenerative disorders of the temporo mandibular joint: etiology, diagnosis, and treatment. J Dent Res. 2008; 87 (4): 296-307.

4. Mazzeto MO, Hotta TH, Mazzetto RG. Analysis of TMJ vibration sounds before and after use of two types of oclusal splints. Braz Dent J. 2009; 20 (4): 325-30.

5. Santander H, Santander MC, Valenzuela S, Fresno MJ, Fuentes A, Gutiérrez MF, et al. Después de cien años de uso: ¿las férulas oclusales tienen algún efecto terapéutico? Rev Clín Per Implant Rehabil Oral. 2011; 4 (1): 29-35.

6. Freese AEM, Picand JLB. Manual práctico de oclusión dentaria. Caracas: Amolca; 2006.

7. Glaros AG, Owais Z, Lausten L. Reduction in parafunctional activity: a potential mechanism for the effectiveness of splint therapy. J Oral Rehabil. 2007; 34 (2): 97-104.

8. Conti PCR, dos Santos CN, Kogawa EM, de Castro Ferreira Conti AC, de Araujo C dos RP. Thetreatment of painful temporo mandibular joint clicking with oral splints: a randomized clinical trial. J Am Dent Assoc. 2006; 137 (8): 1108-14.

9. DuPont JS Jr, Brown CE. Occlusal splints from the beginning to the present. Cranio J Craniomandib Pr. 2006; 24 (2): 141-5.

10. Cummings TM, White A: Needling therapies in the management of miofascial trigger 353 point pain: arthrocentesis systematic review. Arch Phys Med Rehabil. 2001; 82: 986-92.

11. Zonnenberg JM. The efficacy of a specific stabilization splint. The Journal of Craniomandibular \& Sleep Practice. 2014; 32 (1): 68-74.

12. Hanefi KE. Comparison off effectiveness of stabilization splint, anterior repositioning splint and behavioral therapy in treatment of disc displacement with reduction. Departament of Prosthodontics. 2011; 57 (1): 23-30.

13. Niemela K, Korpela M, Raustia A. Efficacy of stabilization splint treatment on temporomandibular disorder. Journal of Oral Rehabilitation. 2012; 39: 799-804.

14. Saavedra J BJCD. Férulas oclusales. Estomatol Herediana 2012; 22 (4).

15. Casares G, Thomas A, Carmona J. Influence of oral stabilization appliances in intra- articular pressure of the temporomandibular joint. The journal of Craniomndibular \& Sleep Practice. 2014; 32 (3). 
16. Hasegawa Y, Kakimoto N, Tomita S, Honda K, Tanaka Y, Yagi K, et al. Movement of the mandibular condyle and articular disc on placement of an occlusal splint. Oral Surg Oral Med Oral Pathology Oral Radiol Endod. 2011; 112: 640-7.

17. Jokstad A, Mo A, Krogstad BS. Clinical comparison between two different splint designs for temporomandibular disorder therapy. Acta Odontology Scand. 2005; 63:218-26.

18. Kurita H, Kurashina K, Baba H, Ohtsuka A, Kotani A, and Kopp S. Evaluation of disk capture with a splint reposition in appliance: clinical and critical assess ment with MR imaging. Oral Surg Oral Med Oral Pathology Oral Radiol Endod. 1998; 85: $377-80$.

19. Kurita $H$, Kurashina K, Ohtsuka A, Kotani A. Change of position of the temporomandibular joint disk with insertion of a disk-reposition in appliance. Oral Surg Oral Med Oral Pathol Oral Radiol Endod. 1998; 85: 142-5.

20. Kurita $H$, Ohtsuka A, Kobayashi $H$, Kurashina K. A study of the relationship between the positions of the condylar head and displacement of the temporomandibular joint disk. Dento maxillary facial Radiol. 2001; 30: 162-5.

21. Kurita H, Uehara S, Yokochi M, Nakatsuka A, Kobayashi H, Kurashina K. A longterm follow-up study of radiographically evident degenerative changes in the temporomandibular joint with different conditions of disk displacement. Int J Oral Maxillofac Surg. 2006; 35 (1): 49-54.

22. Liu MQ, Chen HM, Yap AU, Fu KY. Condylar remodeling accompany ying splint therapy: a cone-beam computerized tomography study of patients with temporomandibular joint disk displacement. Oral Surg Oral Med Oral Pathol Oral Radiol. 2012; 114: 259-65.

23. Moloney F, Howard JA. Internal derangements of the temporomandibular joint. III. Anterior repositioning splint therapy. Aust Dent J. 1986; 31: 30-9.

24. Okeson JP. Management of temporo mandibular disorders and occlusion. 7th ed. Saint Louis: Mosby; 2012.

25. Santacatterina A, Paoli M, Peretta R, Bambace A, Beltrame A. A comparison between horizontal splint and repositioning splint in the treatment of "disc dislocation with reduction". Literature meta-analysis. Oral Rehabil. 1998; 25 (2): 81-8.

26. Simmons HC 3rd, Board of Directors, American Academy of Craniofacial Pain. Guidelines for anterior repositioning appliance therapy for the management of craniofacial pain and TMD. Cranio. 2005; 23: 300-5.

27. Tecco S, Festa F, Salini V, Epifania E, D'Attilio M. Treatment of joint pain and joint noises associated with a recent TMJ internal derangement: a comparison of an anterior repositioning splint, a full arch maxillary stabilization splint, and an untreated control group. Cranio. 2004; 22: 209-19.

28. Wassell RW, Adams N, Kelly PJ. Thetreatment of temporomandibular disorders with stabilizing splints in general dental practice: one-year follow-up. J Am Dent Assoc. 2006; 137: 1089-98. 
29. Whyte AM, McNamara D, Rosenberg I, Whyte AW. Magnetic resonance imaging in the evaluation of temporomandibular joint disc displacement - a review of 144 cases. Int J Oral Maxillofac Surg. 2006; 35: 696-703.

30. Higgins JA, D G, Sterne J. Assessing risk of bias in included studies. In: Higgins J, Altman D, SterneJ (editors). Cochrane handbook for systematic review soft interventions version 5.1.0 [updated March 2011]. The Cochrane Collaboration; 2011.

31. Jadad AR, Moore RA, Carroll D, Jenkinson C, Reynolds DJ, Gavaghan DJ, et al. assessing the quality of reports of randomized clinical trials: is blinding necessary? Control Clin Trials. 1996; 17(1).

32. Rubinoff MS, Gross A, McCall WD Jr. Conventional and no occluding splint therapy compared for patients with myofascial pain dysfunction syndrome. Gen Dent. 1987; 35 (6).

33. Dao TT, Lavigne GJ, Charbonneau A, Feine JS, Lund JP. The efficacy of oral splints in the treatment of miofascial pain of the jaw muscles: a controlled clinical trial. Pain. 1994; 56 (1): 85-94.

34. Ekberg E, Vallon D, Nilner M. Occlusal appliance therapy in patients with temporomandibular disorders A double-blind controlled study in a short-term perspective. Acta Odontol Scand. 1998; 56 (2): 122-8.

35. Ekberg E, Nilner M. A 6-and 12-month follow-up of appliance therapy in TMD patients: A follow-up of acontrolled trial. Int J Prosthodont. 2002; 15 (6): 564-70.

36. Ekberg E, Vallon D, Nilner M. The efficacy of appliance therapy in patients with temporomandibular disorders of mainly genus origin. A randomized, controlled, short-term trial. J Orofac Pain. 2003; 17(2): 133-9.

37. Kuttila M, Le Bell Y, Savolainen-Neimi E, Kuttila S, Alanen P. Efficiency of occlusal appliance therapy in secondary otalgia and temporomandibular disorders. Acta Odontol Scand. 2002; 60 (4): 248-54.

38. Wassell RW, Adams N, Kelly PJ. Treatment of temporomandibular disorders by stabilizing splints in general dental practice: results after initial treatment. British Dental Journal. 2004; 197(1): 1089-98.

39. Wassell RW, Adams N, Kelly PJ. The treatment of temporomandibular disorders with stabilizing splints in general dental practice: one-year follow-up. Journal of the American Dental Association. 2006; 137 (8): 1089-98.

40. Dahlstrom L, Haraldson T. Bite plates and stabilization splints in mandibular dysfunction. A clinical and electromyography comparison. Acta Odontol Scand. 1985; 43 (2): 109-14.

41. Gray RJ, Davies SJ, Quayle AA, Wastell DG. A comparison of two splints in the treatment of TMJ pain dysfunction syndrome. Can occlusal analysis be used to predict success of splint therapy? Br Dent J. 1991; 170 (8): 288.

42. Stiesch-Scholz M, Kempert J, Wolter S, Tschernitschek H, Rossbach A. Comparative prospective study on splint therapy of anterior disc displacement without reduction. J Oral Rehabilitation. 2005; 32 (7): 474-9. 
43. Al Quran FAM, Kamal MS. Anterior midline point stop device (AMPS) in the treatment of myogenous TMDs: Comparison with the stabilization splint and control group. Oral Surgery Oral Medicine Oral Pathology Oral Radiology and Endodontics. 2006; 101 (6): 741-7.

44. Conti PCR, de Alencar EN, da Mota Correa AS, Lauris JRP, Porporatti AL, Costa YM. Behavioural changes and occlusal splints are effective in the management of masticatory miofascial pain: a short-term evaluation. Journal of Oral Rehabilitation. 2012; 39 (10): 754-60.

45. Truelove E, Higgins KH, Mancl L, Dworkin SF. The efficacy of traditional, low-cost and non splint therapies for temporo mandibular disorder: A randomized controlled trial. Journal of the American Dental Association. 2006; 137 (8): 1099-107.

46. Raustia AM, Pohjola RT, Virtanen KK. Acupuncture compared with stomatognathic treatment for TMJ dysfunction. Part I: A randomized study. J Prosthet Dent. 1985; 54 (4): 581-5.

47. Oz S, Gokcen-Rohlig B, Saruhanoglu A, Tuncer EB. Management of myofascial pain: low-level laser therapy versus occlusal splints. Journal of Craniofacial Surgery. 2010; 21 (6): 1722-8.

48. Ismail F, Demling A, Hessling K, Fink M, Stiesch-Scholz M. Short-term efficacy of physical therapy compared to splint therapy in treatment of arthrogenous TMD. Journal of Oral Rehabilitation. 2007; 34 (11): 807-13.

49. Johansson A, Wenneberg B, Wagersten C, Haraldson T. Acupuncture in treatment of facial muscular pain. Acta Odontol Scand. 1991; 49 (3): 153-8.

50. List T, Helkimo M, Karlsson R. Pressure pain thre sholds in patients with craniomndibular disorders before and after treatment with acupuncture and occlusal splint therapy: a controlled clinical study. J Orofac Pain. 1993; 7 (3): 275-82.

51. Carlson CR, Bertrand PM, Ehrlich AD, Maxwell AW, Burton RG. Physical selfregulation training for the management of temporo mandibular disorders. J Orofac Pain. 2001; 15 (1): 47-55.

52. Shedden-Mora MC, Weber D, Neff A, Rief W. Biofeedback-based Cognitive behavioral treatment compared with occlusal splint for temporomandibular disorder a randomized controlled trial. Clinical Journal of Pain. 2013; 29 (12): 1057-65.

53. Turk DC, Zaki HS, Rudy TE. Effects of intraoral appliance and biofeedback/stress management alone and in combination in treating pain and depression in patients with temporo mandibular disorders. J Prosthet Dent. 1993; 70 (2): 158-64.

54. Glaros AG, Kim-Weroha N, Lausten L, Franklin KL. Comparison of habit reversal and a behaviorally-modified dental treatment for temporomandibular disorders: a pilot investigation. Appl Psychophysiology Biofeedback. 2007; 32 (3-4): 149-54.

55. Niemela K, Korpela M, Raustia A, Ylostalo P, Sipila K. Efficacy of stabilization splint treatment on temporomandibular disorders. J Oral Rehabil. 2012; 39 (11): 799-804. 
56. Lundh H, Westesson PL, Eriksson L, Brooks SL. Temporomandibular joint disk displacement without reduction. Treatment with flat occlusal splint versus no treatment. Oral Surg Oral Med Oral Pathol. 1992; 73 (6): 655.

57. Daif ET. Correlation of splint therapy outcome with the electromyography of masticatory muscles in temporomandibular disorder with myofascial pain. Acta Odontológica Scandinavia. 2012; 70 (1): 72-7.

58. Nitecka-Buchta A, Marek B, Baron S. CGRP plasma level changes in patients with temporomandibular disorders treated with occlusal splint - a randomized clinical trial. Endocrinology Polska. 2014; 65 (3): 217-23. 